\title{
THE EQUIVALENCE OF RUBIN'S CONJECTURE AND THE ETNC/LRNC FOR CERTAIN BIQUADRATIC EXTENSIONS
}

\author{
PAUL BUCKINGHAM \\ Department of Mathematical and Statistical Sciences, University of Alberta, \\ Edmonton AB, T6G 2G1, Canada \\ e-mail:p.r.buckingham@ualberta.ca
}

(Received 27 September 2012; revised 1 February 2013; accepted 3 April 2013; first published online 13 August 2013)

\begin{abstract}
For an abelian extension $L / K$ of number fields, the Equivariant Tamagawa Number Conjecture (ETNC) at $s=0$, which is equivalent to the Lifted Root Number Conjecture (LRNC), implies Rubin's Conjecture by work of Burns [3]. We show that, for relative biquadratic extensions $L / K$ satisfying a certain condition on the splitting of places, Rubin's Conjecture in turn implies the ETNC/LRNC. We conclude with some examples.
\end{abstract}

2010 Mathematics Subject Classification. Primary 11R42, Secondary 11R70.

1. Introduction. The Equivariant Tamagawa Number Conjecture (ETNC), formulated by Burns and Flach in [6], is a deep conjecture concerning leading coefficients of Taylor series of $L$-functions associated to motives defined over number fields. We will be interested in the case where the motive in question is the Tate motive $h^{0}(\operatorname{Spec}(L))(0)$ with the natural action by $\mathbb{Z}[\mathrm{Gal}(L / K)]$, where $L / K$ is a Galois extension of number fields. In this setting, the ETNC is equivalent [2, Theorem 2.3.3] to the Lifted Root Number Conjecture (LRNC) of Gruenberg-Ritter-Weiss formulated in [14], and it is this version of the conjecture that we choose to work with in the present paper. In order not to favour either name (ETNC/LRNC) in our discussions, we refer to both conjectures as $\mathrm{Z}(L / K)$.

To put $\mathrm{Z}(L / K)$ in context, it can be viewed as an integral refinement of Stark's Conjecture - see for example [29, Chap. I, Conj. 5.1] and [25]. Thus, in some sense, $\mathrm{Z}(L / K)$ might be considered an analytical class number formula over $\mathbb{Z}[G]$. However, there is another (and earlier) integral refinement of Stark's Conjecture, namely Rubin's Conjecture [22, Conj. B]. Burns showed in [3, Cor. 9.2] that $\mathrm{Z}(L / K)$ implies Rubin's Conjecture. Our aim in this paper is to show that, for certain (relative) biquadratic extensions, the reverse implication holds. This will need to be made more precise, since $\mathrm{Z}(L / K)$ depends only on the extension, while Rubin's Conjecture takes extra data as input. The exact statement can be found in Theorem 8.3.

We briefly remark on the type of biquadratic extension $L / K$ to which our results apply: We assume that all infinite places split completely, only one place ramifies, and the ramified place has full decomposition group. This condition will be rephrased in Lemma 8.1. Biquadratic extensions of this type exist. We conclude with some examples, two of which are concrete ones in which $\mathrm{Z}(L / K)$ is shown to hold (see Section 11.3). These examples do not follow from previously known results.

Concerning work already in the literature, we highlight in particular the papers of Dummit-Sands-Tangedal [10], Sands [23] and Macias Castillo [17], since they all 
deal with multi-quadratic extensions. The first proves, under certain assumptions, the integral Stark conjecture for $L$-functions whose order of vanishing is 1 at $s=0$, in other words, Rubin's Conjecture when $r=1$ (see Section 3). The second deals with a slight weakening of Rubin's Conjecture, referred to as Popescu's Conjecture; see [20] for a formulation. The third paper proves results concerning the annihilation of classgroups using derivatives of $L$-functions, cleverly avoiding having to prove $\mathrm{Z}(L / K)$ in full.

2. $L$-functions and basic notation. Let $L / K$ be a Galois extension of number fields and $S$ a finite set of places of $K$ containing the infinite ones. We assume knowledge of the definition of the $S$-truncated Artin $L$-function $L_{L / K, S}(s, \chi)$ associated to a character $\chi$ of the Galois group $G$ of $L / K$. For example, see [29, Chap. 0, Section 4 and Chap. I, Section 3].

We will also need the $(S, T)$-modified $L$-function $L_{L / K, S, T}(s, \chi)$, where $T$ is a finite set of places of $K$ that is disjoint from $S$. To define it, we assume for simplicity that $L / K$ is abelian and that $S$ contains the ramified places (this will be assumed throughout the paper) so that $T$ necessarily consists of unramified places. Then for $\chi \in \widehat{G}=\operatorname{Hom}_{\mathbb{Z}}\left(G, \mathbb{C}^{\times}\right)$,

$$
L_{L / K, S, T}(s, \chi)=L_{L / K, S}(s, \chi) \prod_{\mathfrak{p} \in T}\left(1-\chi\left(\operatorname{Fr}_{\mathfrak{p}}\right) \mathbf{N p}^{1-s}\right)
$$

where $\operatorname{Fr}_{\mathfrak{p}}$ denotes the arithmetic Frobenius at $\mathfrak{p}$. In the case where $\chi$ is the trivial character $\mathbf{1}$, we will set $\zeta_{K, S}(s)=L_{L / K, S}(s, \mathbf{1})$ and $\zeta_{K, S, T}(s)=L_{L / K, S, T}(s, \mathbf{1})$. For any complex function $f(s)$ that is analytic at the point $s_{0} \in \mathbb{C}, f^{*}\left(s_{0}\right)$ will denote the leading coefficient of the Taylor series of $f(s)$ at $s=s_{0}$, and $f^{(r)}\left(s_{0}\right)$ will denote the $r$ th derivative at $s=s_{0}$ for each $r \geq 0$.

2.1. Notation. Let $S$ be a finite set of places of $K$ containing the infinite ones and $T$ a finite set of places of $K$ disjoint from $S$. We will use the following notation:

$$
\begin{aligned}
S_{L}, T_{L}: & \text { The set of places of } L \text { above those in } S \text { and } T \text { respectively } \\
\mathrm{Cl}_{S}(L): & \text { The } S_{L} \text {-class-group of } L \text { (or simply } S \text {-class-group) } \\
\mathrm{Cl}_{S, T}(L): & \text { The }(S, T) \text {-class-group of } L, \text { i.e. the quotient of the group } \\
& \text { of fractional ideals of } \mathcal{O}_{L, S} \text { prime to } T_{L} \text { by the subgroup of } \\
& \text { principal ideals } a \mathcal{O}_{L, S} \text { with } a \equiv 1 \text { mod } \mathfrak{P} \text { for all places } \mathfrak{P} \in T_{L} \\
U_{S}: & \text { The group } \mathcal{O}_{L, S}^{\times} \text {of } S_{L} \text {-units in } L \text { (or simply } S \text {-units) } \\
U_{S, T}: & \text { The group of }(S, T) \text {-units in } L \text { (see }(3.1) \text { ) } \\
G_{v}: & \text { The decomposition group of the place } v \text { of } K \text { in } G= \\
& \text { Gal }(L / K) \text { when } L / K \text { is abelian } \\
\widehat{G}: & \text { Hom }\left(G, \mathbb{C}^{\times}\right) \text {if } G \text { is abelian } \\
e_{\chi}: & \text { The idempotent in } \mathbb{C}[G] \text { of the character } \chi \in \widehat{G} \\
Y_{S}: & \text { The free abelian group on } S_{L} \\
X_{S}: & \text { The kernel of the augmentation map } Y_{S} \rightarrow \mathbb{Z} \\
\simeq \quad: \text { Canonical isomorphism } & \text { Non-canonical isomorphism }
\end{aligned}
$$


3. Rubin's conjecture. We now turn to the formulation of Rubin's Conjecture. Background and results on this conjecture can be found in the papers by Greither [13], Popescu [20, 21] and Vallières [30].

Before stating the conjecture, we need to introduce some notation. As explained in [22, Section 1.2], if $M$ is a $\mathbb{Z}[G]$-module (where $G$ is an arbitrary finite abelian group for the time being), then for each $r \geq 0$ there is a well-defined homomorphism

$$
\begin{aligned}
\bigwedge_{\mathbb{Z}[G]}^{r} \operatorname{Hom}_{\mathbb{Z}[G]}(M, \mathbb{Z}[G]) \rightarrow \operatorname{Hom}_{\mathbb{Z}[G]}\left(\bigwedge_{\mathbb{Z}[G]}^{r} M, \mathbb{Z}[G]\right) \\
\phi_{1} \wedge \cdots \wedge \phi_{r} \mapsto\left(m_{1} \wedge \cdots \wedge m_{r} \mapsto \operatorname{det}\left(\phi_{i}\left(m_{j}\right)\right)\right) .
\end{aligned}
$$

By abuse of notation, we will denote the image of $\phi_{1} \wedge \cdots \wedge \phi_{r}$ under this map by the same symbol so that given $m_{1}, \ldots, m_{r} \in M$ we write simply

$$
\left(\phi_{1} \wedge \cdots \wedge \phi_{r}\right)\left(m_{1} \wedge \cdots \wedge m_{r}\right)=\operatorname{det}\left(\phi_{i}\left(m_{j}\right)\right)
$$

We will also extend the $\operatorname{map}\left(\phi_{1} \wedge \cdots \wedge \phi_{r}\right)(-)$ linearly to $\mathbb{Q} \otimes_{\mathbb{Z}}\left(\bigwedge_{\mathbb{Z}[G]}^{r} M\right) \rightarrow \mathbb{Q}[G]$.

Definition 3.1. For any $\mathbb{Z}[G]$-module $M$ and any $r \geq 0$, define $\bigwedge_{0}^{r} M$ to be

$$
\left\{m \in \mathbb{Q} \otimes_{\mathbb{Z}}\left(\bigwedge_{\mathbb{Z}[G]}^{r} M\right) \mid\left(\phi_{1} \wedge \cdots \wedge \phi_{r}\right)(m) \in \mathbb{Z}[G] \text { for all } \phi_{i} \in \operatorname{Hom}_{\mathbb{Z}[G]}(M, \mathbb{Z}[G])\right\} .
$$

Now suppose $G$ is the Galois group of an abelian extension $L / K$ of number fields. We will once again take a finite set $S$ of places of $K$ containing the infinite and ramified ones, and a finite set $T$ of places of $K$, disjoint from $S$. For a character $\chi$ of $G$, we let $r(\chi)=\left\langle\chi, \mathbb{C} \otimes_{\mathbb{Z}} U_{S}\right\rangle$, which is also the order of vanishing of the $L$-function $L_{L / K, S}(s, \chi)$ at $s=0$ by [29, Chap. I, Prop. 3.4]. (Note that $r(\chi)$ depends only on $S$, not $T$.) Then set

$$
U_{S, T}=\left\{u \in U_{S} \mid u \equiv 1 \bmod \mathfrak{P} \text { for all places } \mathfrak{P} \in T_{L}\right\}
$$

and

$$
\Omega_{S, T, r}=\left\{u \in \bigwedge_{0}^{r} U_{S, T} \mid e_{\chi} u=0 \text { for all } \chi \in \widehat{G} \text { with } r(\chi) \neq r\right\}
$$

We assume the following hypotheses.

(St1) $S$ contains the infinite and ramified places.

(St2) $S$ contains at least $r$ places that split completely in $L / K$.

(St3) $S$ contains at least $r+1$ places.

(St4) $U_{S, T}$ is $\mathbb{Z}$-torsion-free.

We point out that (St4) is satisfied in particular if $T$ contains at least two places of different residue characteristics. The hypotheses $(\mathbf{S t} 2)$ and $(\mathbf{S t} 3)$ ensure that the order of vanishing of $L_{L / K, S, T}(s, \chi)$ at $s=0$ is at least $r$ for all $\chi \in \widehat{G}$; see [29, Chap. I, Prop. 3.4]. (Note, however, the comment concerning the conjecture of Emmons and Popescu [11] in Section 3.1.)

Definition 3.2. (i) A triple $(S, T, r)$ satisfying hypotheses $(\mathbf{S t} 1)$ to $(\mathbf{S t} 4)$ is called a Rubin datum for the extension $L / K$. 
(ii) If $(S, T, r)$ is a Rubin datum for $L / K$, let

$$
\theta_{L / K, S, T}^{(r)}=\sum_{\chi \in \widehat{G}} \frac{1}{r !} L_{L / K, S, T}^{(r)}(0, \bar{\chi}) e_{\chi} .
$$

We have introduced the term 'Rubin datum' purely to make various statements in the paper more succinct.

Consider the regulator map $\lambda: \mathbb{C} \otimes_{\mathbb{Z}} U_{S} \rightarrow \mathbb{C} \otimes_{\mathbb{Z}} X_{S}$ given by

$$
1 \otimes u \mapsto-\sum_{w \in S_{L}} \log \|u\|_{w} \cdot w
$$

REMARK 3.3. The map $\lambda$ in (3.3) differs from the usual Dirichlet regulator map by a sign. This makes no difference to the formulation of Rubin's Conjecture but is crucial for $\mathrm{Z}(L / K)$.

Denote by $\lambda^{(r)}: \mathbb{R} \otimes_{\mathbb{Z}}\left(\bigwedge_{\mathbb{Z}[G]}^{r} U_{S}\right) \rightarrow \mathbb{R} \otimes_{\mathbb{Z}}\left(\bigwedge_{\mathbb{Z}[G]}^{r} X_{S}\right)$ the map induced by $\lambda$. Also, if $A$ is a subring of $\mathbb{C}$ and $M$ is an $A[G]$-module, write $\bigwedge_{A[G] \text {,tf }}^{r} M$ for the image of $\bigwedge_{A[G]}^{r} M$ in $\mathbb{C} \otimes_{A}\left(\bigwedge_{A[G]}^{r} M\right)$. The following is [22, Conjecture B], which we refer to as $\mathrm{B}(L / K, S, T, r)$. We will also refer to it as Rubin's Conjecture when the quadruple $(L / K, S, T, r)$ is understood or not important.

CONJECTURe 3.4. If $(S, T, r)$ is a Rubin datum for $L / K$, then

$$
\theta_{L / K, S, T}^{(r)} \bigwedge_{\mathbb{Z}[G], \mathrm{tf}}^{r} X_{S} \subseteq \lambda^{(r)}\left(\Omega_{S, T, r}\right)
$$

3.1. Known cases of Rubin's Conjecture. Rubin's Conjecture is known in the following cases:

(i) All extensions $L / K$ such that $L / \mathbb{Q}$ is abelian.

(ii) $K$ contains an imaginary quadratic field $k$ of class number one such that $L / k$ is abelian and $[L: K]$ is odd and divisible only by rational primes that split in $k / \mathbb{Q}$.

(iii) All abelian extensions $L / K$, where $K$ is an arbitrary imaginary quadratic field, in the case $r=1$.

(iv) Arbitrary quadratic extensions $L / K$.

(v) A large class of multi-quadratic extensions, in the case $r=1$.

(vi) char $(K)>0$ (Rubin's Conjecture may be formulated equally well in positive characteristic; see, for example, [19]).

Recall that, by Burns [3, Cor. 9.2], Z( $L / K)$ implies Rubin's Conjecture for all Rubin data $(S, T, r)$ for $L / K$. Therefore, cases (i) and (ii) above follow from the truth of $\mathrm{Z}(L / K)$ for $L / K$ in the specified situations: (i) by Burns-Greither [7] and Flach [12], and (ii) by Bley [1].

Case (iii) was proven in [25], where Stark formulated a conjecture that was later generalized by Rubin to what we now call Rubin's Conjecture. See also [29, Chap. IV, Prop. 3.9].

Case (iv) was proven in [22] itself. As for case (v), the known cases of Rubin's Conjecture for multi-quadratic extensions are due principally to Dummit-Sands-Tangedal 
[10]; we refer the reader to that paper for a precise description of the assumptions made on $L / K$.

Case (vi) in the above list was done in part by Popescu - see [20] - and completed for arbitrary global function fields by Burns in [4]. In fact, [4] establishes the truth of a stronger conjecture in the positive characteristic case, one that is analogous to $\mathrm{Z}(L / K)$.

It is also worth pointing out that Emmons and Popescu [11] have formulated a version of Rubin's Conjecture that weakens the hypotheses (St1) to (St4). Vallières [30] has shown that the conjecture of Emmens and Popescu follows from $\mathrm{Z}(L / K)$, and so it holds in particular in cases (i) and (ii) above.

4. $K$-theory: definitions. Let $G$ be a finite group. We will work with lowdimensional $K$-groups for rings $R$ equal to $\mathbb{Z}[G], \mathbb{Q}[G]$, or a maximal order $\mathcal{M}$ in $\mathbb{Q}[G]$ containing $\mathbb{Z}[G]$. Certain aspects of the theory (even the definition of $K_{1}$ ) can be made simpler by assuming right away that $G$ is abelian, which we will therefore do.

$K_{0}(R)$ : The Grothendieck group of the category of finitely generated, projective $R$-modules.

$K_{1}(R) \quad$ : For $R$ as above, we may take this to be the group of units $R^{\times}$ in $R$.

$K_{0}(R, F) \quad$ : The relative $K_{0}$-group of $R$ with coefficients in $F$, where $F$ is equal to $\mathbb{Q}$ or $\mathbb{R}$ and $R$ is equal to $\mathbb{Z}[G]$ or $\mathcal{M}$.

When $F=\mathbb{Q}$ or $\mathbb{R}$ and $R=\mathbb{Z}[G]$ or $\mathcal{M}, K_{0}(R, F)$ is generated by triples $(P, f, Q)$, where $P, Q$ are finitely generated, projective $R$-modules and $f: F[G] \otimes_{R} P \rightarrow$ $F[G] \otimes_{R} Q$ is an $F[G]$-module isomorphism, subject to certain relations which can be found in [26, p. 215]. In the case $F=\mathbb{Q}$ and $R=\mathbb{Z}[G]$, we will be more interested in the description of $K_{0}(\mathbb{Z}[G], \mathbb{Q})$ as the Grothendieck group of finite, cohomologically trivial $\mathbb{Z}[G]$-modules: if $C$ is finite and cohomologically trivial, then it has a projective resolution of the form $0 \rightarrow P \stackrel{f}{\rightarrow} Q \rightarrow C \rightarrow 0$, and we identify the class of $C$ with the element $(P, 1 \otimes f, Q)$ of $K_{0}(\mathbb{Z}[G], \mathbb{Q})$, where $1 \otimes f: \mathbb{Q} \otimes_{\mathbb{Z}} P \rightarrow \mathbb{Q} \otimes_{\mathbb{Z}} Q$ is the isomorphism arising from $f$.

There is a localization sequence

$$
K_{1}(\mathbb{Z}[G]) \rightarrow K_{1}(\mathbb{Q}[G]) \rightarrow K_{0}(\mathbb{Z}[G], \mathbb{Q}) \rightarrow K_{0}(\mathbb{Z}[G]) \rightarrow K_{0}(\mathbb{Q}[G])
$$

in $K$-theory (see $[9,(40.9)])$. With the above description of $K_{0}(\mathbb{Z}[G], \mathbb{Q})$, the map $K_{0}(\mathbb{Z}[G], \mathbb{Q}) \rightarrow K_{0}(\mathbb{Z}[G])$ sends the class of the finite, cohomologically trivial module $C$ to $[Q]-[P]$, where $0 \rightarrow P \rightarrow Q \rightarrow C \rightarrow 0$ is exact. The map $K_{1}(\mathbb{Q}[G]) \rightarrow K_{0}(\mathbb{Z}[G], \mathbb{Q})$ sends an element of $\mathbb{Q}[G]^{\times} \cap \mathbb{Z}[G]$ to the class of the finite, cohomologically trivial module $\mathbb{Z}[G] / \alpha \mathbb{Z}[G]$. This describes the map completely, since every element of $\mathbb{Q}[G]^{\times}$ is a quotient of elements of $\mathbb{Q}[G]^{\times} \cap \mathbb{Z}[G]$.

We will need two subgroups of $K_{0}(\mathbb{Z}[G])$, namely the locally free class-group, $\mathrm{Cl}(\mathbb{Z}[G])$, and the $D$-subgroup, $D(\mathbb{Z}[G])$. To define the former, we observe that there is a rank function $\mathrm{rk}_{\mathbb{Z}[G]}: K_{0}(\mathbb{Z}[G]) \rightarrow \mathbb{Z}$, which takes the class of a finitely generated, $\mathbb{Z}[G]$-projective module $M$ to $\frac{1}{|G|} \operatorname{rk}_{\mathbb{Z}}(M)$, an integer. One defines $\mathrm{Cl}(\mathbb{Z}[G])$ to be the kernel of $\mathrm{rk}_{\mathbb{Z}[G]}$; it is a finite group. The $D$-subgroup is defined to be the kernel of $K_{0}(\mathbb{Z}[G]) \rightarrow K_{0}(\mathcal{M})$, and in fact lies in $\mathrm{Cl}(\mathbb{Z}[G])$. This definition is independent of the choice of $\mathcal{M}$. See [32, Chap. 4] for more details. 
5. The conjecture $\mathrm{Z}(L / K)$ for abelian extensions. We emphasize that $\mathrm{Z}(L / K)$ can be formulated for arbitrary Galois extensions of number fields. However, we choose to deal only with abelian extensions, since the statement of the conjecture can be reached more quickly in this case.

Let $G=\operatorname{Gal}(L / K)$, where $L / K$ is abelian. We assume that $S$ is a finite set of places of $K$ containing the infinite and ramified ones, and such that the $S$-class-group $\mathrm{Cl}_{S}(L)$ is zero. We choose a Tate sequence [28]

$$
0 \rightarrow U_{S} \rightarrow A \rightarrow B \rightarrow X_{S} \rightarrow 0
$$

for $(L / K, S)$, i.e. a representative of the Tate canonical class in $\operatorname{Ext}_{\mathbb{Z}[G]}^{2}\left(X_{S}, U_{S}\right)$ with $A$ finitely generated and cohomologically trivial, and $B$ finitely generated and projective. Break the Tate sequence into short exact sequences

$$
0 \rightarrow U_{S} \rightarrow A \rightarrow C \rightarrow 0
$$

and

$$
0 \rightarrow C \rightarrow B \rightarrow X_{S} \rightarrow 0
$$

and choose injective maps $\alpha, \beta: C \rightarrow C$ that are homotopic to zero. (A $\mathbb{Z}[G]$-module homomorphism is said to be homotopic to zero if it factors through a projective module.) With $\alpha$ and $\beta$ thus chosen, the pull-back and push-out of (5.2) and (5.3) along $\alpha$ and $\beta$, respectively, are split. Indeed, if $\alpha$ and $\beta$ factor through the projectives $P$ and $Q$ respectively, then the push-out map factors through $\operatorname{Ext}_{\mathbb{Z}[G]}^{1}\left(P, U_{S}\right)$ and the pull-back map factors through $\operatorname{Ext}_{\mathbb{Z}[G]}^{1}\left(X_{S}, Q\right)$. Both of these Ext-groups vanish, and so the resulting sequences split. (See [31, Section 3.4] for more on push-outs and pull-backs.) Thus, we have maps $\tilde{\alpha}$ and $\tilde{\beta}$ making the following diagrams commute:
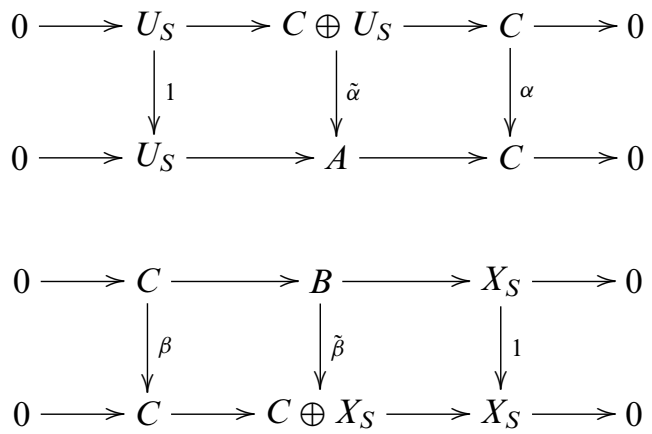

Choose a $\mathbb{Z}[G]$-embedding $\varphi: X_{S} \rightarrow U_{S}$. (Such a $\varphi$ always exists: $\mathbb{Q} \otimes_{\mathbb{Z}} X_{S} \cong \mathbb{Q} \otimes_{\mathbb{Z}} U_{S}$ by [24, Section 12.1], and this can be made into an embedding $X_{S} \rightarrow U_{S}$ by multiplying by a suitable positive integer.) We then let $\Phi: B \rightarrow A$ be the embedding $\tilde{\alpha} \circ(1 \oplus \varphi) \circ \tilde{\beta}$. Since $\operatorname{Coker}(\Phi)$ is finite ( $A$ and $B$ have the same $\mathbb{Z}$-rank) and cohomologically trivial, it therefore defines an element of $K_{0}(\mathbb{Z}[G], \mathbb{Q})$, which we shall denote $\Delta(\varphi, \alpha, \beta)$ to reflect its dependence on $\varphi, \alpha$ and $\beta$.

One can compensate for the choice of $\alpha$ and $\beta$ above by letting $\Xi(\alpha, \beta)$ be the image, under $K_{1}(\mathbb{Q}[G]) \rightarrow K_{0}(\mathbb{Z}[G], \mathbb{Q})$, of the $\mathbb{Q}[G]$-determinant of the automorphism $\alpha \circ \beta: \mathbb{Q} \otimes_{\mathbb{Z}} C \rightarrow \mathbb{Q} \otimes_{\mathbb{Z}} C$. The element $\Delta(\varphi, \alpha, \beta)-\Xi(\alpha, \beta)$ does not depend on $\alpha$, $\beta, \tilde{\alpha}$ or $\tilde{\beta}[\mathbf{1 4}$, Lemma 1]. 
If $\chi$ is a character of $G$ and $V_{\bar{\chi}}$ is a $\mathbb{C}[G]$-module with character $\bar{\chi}$, then we let $R_{\varphi}(\chi)$ be the determinant over $\mathbb{C}$ of the $\mathbb{C}$-automorphism of $\operatorname{Hom}_{\mathbb{C}[G]}\left(V_{\bar{\chi}}, \mathbb{C} \otimes_{\mathbb{Z}} X_{S}\right)$ given by $h \mapsto \lambda \circ \varphi \circ h$, where $\lambda$ is the regulator map defined in (3.3). Then let $A_{\varphi}(\chi)=$ $R_{\varphi}(\chi) / L_{L / K, S}^{*}(0, \chi) \in \mathbb{C}^{\times}$, and consider the map

$$
\begin{aligned}
R(G) & \rightarrow \mathbb{C}^{\times} \\
\chi & \mapsto A_{\varphi}(\chi),
\end{aligned}
$$

where $R(G)$ is the representation ring of $G$. Stark's Conjecture [29, Chap. I, Conj. $5.1]$ is the assertion that this map in fact lies in the subgroup $\operatorname{Hom}_{\Gamma}\left(R(G),\left(\mathbb{Q}^{\mathrm{c}}\right)^{\times}\right)$of $\operatorname{Hom}\left(R(G), \mathbb{C}^{\times}\right)$, where $\mathbb{Q}^{\mathrm{c}}$ is the algebraic closure of $\mathbb{Q}$ in $\mathbb{C}$ and $\Gamma=\operatorname{Gal}\left(\mathbb{Q}^{\mathrm{c}} / \mathbb{Q}\right)$. Let us now assume Stark's Conjecture. Then the element

$$
\mathcal{A}_{\varphi}=\sum_{\chi \in \widehat{G}} A_{\varphi}(\bar{\chi}) e_{\chi}
$$

in fact lies in $\mathbb{Q}[G]^{\times}$. We now define $\Upsilon(\varphi)$ to be the image under $K_{1}(\mathbb{Q}[G]) \rightarrow$ $K_{0}(\mathbb{Z}[G], \mathbb{Q})$ of the class of $\mathcal{A}_{\varphi}$ in $\mathbb{Q}[G]^{\times} / \mathbb{Z}[G]^{\times}$.

Finally, define $\omega(L / K)=\Upsilon(\varphi)-\Delta(\varphi, \alpha, \beta)+\Xi(\alpha, \beta) \in K_{0}(\mathbb{Z}[G], \mathbb{Q})$. By $\quad[\mathbf{1 4}$, Theorem 2'], $\omega(L / K)$ depends only on the extension $L / K$. In particular, it does not depend on the choice of the set $S$, nor on $\varphi, C, \alpha, \beta, \tilde{\alpha}$, or $\tilde{\beta}$.

Conjecture $\mathrm{Z}(L / K)$, in its formulation as the LRNC [14, Section 7], is as follows.

Conjecture 5.1. L/K satisfies Stark's Conjecture and $\omega(L / K)=0$.

$\mathrm{Z}(L / K)$ is known in cases (i), (ii) and (iv) of Section 3.1, and the positivecharacteristic analogue is known in case (vi) of that section. The papers in which the proofs of these cases appeared were discussed in Section 3.1, with the exception of case (iv). In that case, a proof can be found in Kim's PhD thesis [16], but no proof has been published before now. We will provide a short proof in Section 7.

We also point out a recent paper of Johnston and Nickel [15], which provides (among other things) some new non-abelian cases of the conjecture.

Some explicit examples will be given at the end of this paper in the case where $K$ is an imaginary quadratic field. These examples are not already covered by Bley's work [1], since the extensions are assumed to be of odd degree there, and that is not the case for us.

5.1. Related conjectures. There are two related conjectures that deserve to be mentioned. First, there is the Strong Stark Conjecture, which is the assertion that

(a) Stark's conjecture holds for the extension $L / K$,

and (b) the image of $\omega(L / K)$ in $K_{0}(\mathcal{M}, \mathbb{Q})$ is zero.

This is a reformulation (see [2, Theorem 2.2.4]) of Chinburg's original conjecture [8]. The Strong Stark Conjecture was proven for rational characters in [29, Chap. II, Théorème 6.8]. We will use this fact in the proofs of Propositions 7.2 and 10.5. Note that property (b) is equivalent to $\omega(L / K)$ having finite order in $K_{0}(\mathbb{Z}[G], \mathbb{Q})$.

Second, there is Chinburg's third $\Omega$-conjecture [8]. Under our assumptions (namely, that $L / K$ is abelian), this is the assertion that $[A]-[B]=0$ in $K_{0}(\mathbb{Z}[G]$ ), in the notation of Section 5. Put another way, it asserts that the image of $\omega(L / K)$ in 
$K_{0}(\mathbb{Z}[G])$ is trivial. It is this interpretation that inspired the modifier 'lifted' in LRNC, since in some sense $Z(L / K)$ 'lifts' Chinburg's third $\Omega$-conjecture - also known as the root number conjecture due to its relationship to Artin root numbers in the general case - to $K_{0}(\mathbb{Z}[G], \mathbb{Q})$.

6. $K$-theory: lemmas. Although our definitions in Section 4 of the lowdimensional $K$-groups were made only in the case where $G$ is abelian, both Lemmas 6.1 and 6.2 hold in more generality, and we have chosen to state them in that greater generality.

Let $G$ be a finite group, and choose a maximal order $\mathcal{M}$ in $\mathbb{Q}[G]$ containing $\mathbb{Z}[G]$. Recall that $D(\mathbb{Z}[G])$ is the kernel of $K_{0}(\mathbb{Z}[G]) \rightarrow K_{0}(\mathcal{M})$.

Consider the diagram

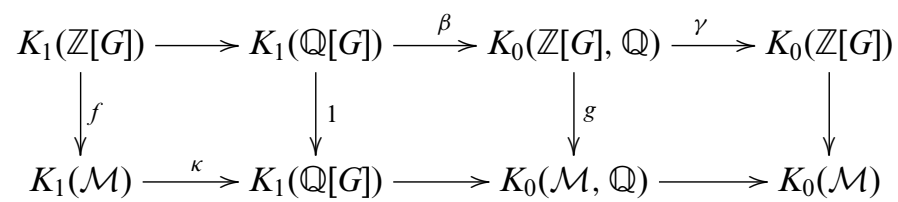

Lemma 6.1. Assume that $\kappa: K_{1}(\mathcal{M}) \rightarrow K_{1}(\mathbb{Q}[G])$ is injective, which is the case if $G$ is abelian. Then $\beta \circ \kappa$ defines an injective map $\operatorname{Coker}(f) \rightarrow \operatorname{Ker}(g)$. If $D(\mathbb{Z}[G])=0$, then this map is an isomorphism.

Proof. Apply the Snake Lemma to the diagram

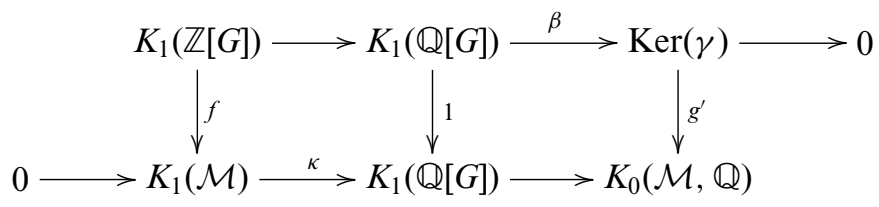

to obtain an isomorphism $\operatorname{Coker}(f) \rightarrow \operatorname{Ker}\left(g^{\prime}\right)$, where $g^{\prime}$ is the restriction of $g$ to $\operatorname{Ker}(\gamma)$. One sees from the proof of the Snake Lemma that this isomorphism is given by $\beta \circ \kappa$. Since $\operatorname{Ker}\left(g^{\prime}\right) \subseteq \operatorname{Ker}(g)$, this gives the desired embedding $\operatorname{Coker}(f) \rightarrow \operatorname{Ker}(g)$.

Finally, if $D(\mathbb{Z}[G])=0$ then $\operatorname{Ker}\left(g^{\prime}\right)=\operatorname{Ker}(g)$, completing the proof.

LEMMA 6.2. Assume that $\mathbb{Q}[G]$ has no simple component that is a totally definite quaternion algebra (for example, if $G$ is abelian). If $X$ and $Y$ are finitely generated, projective $\mathbb{Z}[G]$-modules, then $[X]=[Y]$ in $K_{0}(\mathbb{Z}[G])$ if and only if $X \cong Y$.

Proof. Suppose that $[X]=[Y]$ in $K_{0}(\mathbb{Z}[G])$. Then since $X$ and $Y$ are $\mathbb{Z}$-torsionfree, [32, Lemma 9, p. 34] implies that $X$ and $Y$ are stably isomorphic, i.e. there is a finitely generated, projective $\mathbb{Z}[G]$-module $P$ such that $X \oplus P \cong Y \oplus P$. However, the paragraph following the proof of [27, Theorem 9.9] shows that this implies that $X \cong Y$, under our assumption on $G$.

Lemma 6.3. Suppose $G$ has order 4 and exponent 2. Then the following hold.

(i) $\mathrm{Cl}(\mathbb{Z}[G])=0$.

(ii) A finitely generated $\mathbb{Z}[G]$-module is free if and only if it is projective.

(iii) $\mathbb{Z}[G]^{\times}=\{ \pm \sigma \mid \sigma \in G\}$. 
(iv) The maximal order $\mathcal{M}$ of $\mathbb{Q}[G]$ containing $\mathbb{Z}[G]$ is canonically isomorphic to $\bigoplus_{x \in \widehat{G}} \mathbb{Z}$

(v) If $a_{\chi} \in \mathbb{Z}^{\times}$for all $\chi \in \widehat{G}$, then $\sum_{\chi} a_{\chi} e_{\chi} \in \mathbb{Z}[G]^{\times}$if and only if $\left\{\chi \in \widehat{G} \mid a_{\chi}=1\right\}$ has even cardinality.

Proof. (i) See [9, (50.17) (iii)].

(ii) Let $X$ be a finitely generated, projective $\mathbb{Z}[G]$-module, and let $r$ be its rank over $\mathbb{Z}[G]$. Then $[X]-\left[\mathbb{Z}[G]^{r}\right] \in \mathrm{Cl}(\mathbb{Z}[G])$, so $[X]=\left[\mathbb{Z}[G]^{r}\right]$ in $K_{0}(\mathbb{Z}[G])$ by part (i). Therefore, $X \cong \mathbb{Z}[G]^{r}$ by Lemma 6.2.

(iii) Straightforward.

(iv) Each of the characters $\chi \in \widehat{G}$ takes its values in $\mathbb{Z}$, so $\mathcal{M}=\bigoplus_{\chi \in \widehat{G}} \mathbb{Z} e_{\chi} \simeq$ $\bigoplus_{\chi \in \widehat{G}} \mathbb{Z}$.

(v) Take $\sigma \in G$ and write $\sigma=\sum_{\chi \in \widehat{G}} a_{\chi} e_{\chi}$. Then $a_{\chi}=\chi(\sigma)$, so $\left\{\chi \in \widehat{G} \mid a_{\chi}=1\right\}$ has even cardinality since it is the kernel of the map $\widehat{G} \rightarrow\{ \pm 1\}, \chi \mapsto \chi(\sigma)$. The analogous statement for $\sigma$ replaced by $-\sigma$ follows. The converse uses the same ideas.

7. $\mathrm{Z}(L / K)$ for quadratic extensions. In [16, Section 2.4, Rem. i)], Kim proved $\mathrm{Z}(L / K)$ for quadratic extensions. The present author does not know what techniques were used in Kim's proof. However, we feel that it is instructive to provide a proof here, especially because Kim's proof is unpublished and no argument has yet been published to the author's best knowledge. In any case, our proof is short.

LEMMA 7.1. If $G$ has order 2 , then $K_{0}(\mathbb{Z}[G], \mathbb{Q}) \rightarrow K_{0}(\mathcal{M}, \mathbb{Q})$ is injective.

Proof. By $[9,(50.16)$ (iii)], we have $D(\mathbb{Z}[G])=0$ for groups of order 2. Hence, given Lemma 6.1 , it is enough to show that $K_{1}(\mathbb{Z}[G]) \rightarrow K_{1}(\mathcal{M})$ is surjective in this case. However, $K_{1}(\mathbb{Z}[G]) \rightarrow K_{1}(\mathcal{M})$ is injective, and both $K_{1}(\mathbb{Z}[G])$ and $K_{1}(\mathcal{M})$ have order 4 , so we are done. (Note that $K_{1}(\mathbb{Z}[G])=\{ \pm \sigma \mid \sigma \in G\}$ and that $K_{1}(\mathcal{M})$ consists of the four elements $a_{\mathbf{1}} e_{\mathbf{1}}+a_{\chi} e_{\chi}$ where $\widehat{G}=\{\mathbf{1}, \chi\}$ and $a_{\mathbf{1}}, a_{\chi} \in\{-1,1\}$.)

Proposition 7.2. If $L / K$ is quadratic, then $\mathrm{Z}(L / K)$ holds.

Proof. Recall from Section 5.1 that $L / K$ satisfies the Strong Stark Conjecture (since the characters of $G$ are rational), i.e. $\omega(L / K)$ is in the kernel of $K_{0}(\mathbb{Z}[G], \mathbb{Q}) \rightarrow$ $K_{0}(\mathcal{M}, \mathbb{Q})$. Now apply Lemma 7.1.

8. A condition on the set $S$. In this section, we formulate our main theorem. Let us assume that $L / K$ is a biquadratic extension of number fields.

Let $S$ be a non-empty, finite set of places of $K$, and let $r$ be a positive integer. We say that the set $S$ satisfies hypothesis $P_{r}$ if it contains exactly one place with full decomposition group, $r$ places that split completely, and no other places.

LEMMA 8.1. The following are equivalent:

(i) S satisfies hypothesis $P_{r}$.

(ii) $X_{S}$ is free over $\mathbb{Z}[G]$ and $\operatorname{rk}_{\mathbb{Z}[G]}\left(X_{S}\right)=r$.

(iii) $X_{S}$ is projective over $\mathbb{Z}[G]$ and $\mathrm{rk}_{\mathbb{Z}[G]}\left(X_{S}\right)=r$.

(iv) $L_{L / K, S}(s, \chi)$ has order of vanishing $r$ at $s=0$ for every $\chi \in \widehat{G}$. 
Proof. (ii) and (iii) are equivalent by Lemma 6.3. Assuming (i), and letting $\mathfrak{p}$ be the place that has full decomposition group and $\mathfrak{P}$ the place above it, we have

$$
X_{S}=\bigoplus_{\mathfrak{q} \in S \backslash\{\mathfrak{p}\}} \bigoplus_{\mathfrak{Q} \mid \mathfrak{q}} \mathbb{Z}(\mathfrak{Q}-\mathfrak{P})
$$

Since $\mathfrak{q}$ splits completely for $\mathfrak{q} \in S \backslash\{\mathfrak{p}\}, \bigoplus_{\mathfrak{Q} \mid \mathfrak{q}} \mathbb{Z}(\mathfrak{Q}-\mathfrak{P})$ is free over $\mathbb{Z}[G]$ of $\mathbb{Z}[G]$ rank 1 , so (ii) follows.

Now assume that (ii) holds. Recalling $Y_{S}$ from Section 2.1, we see from the exact sequence

$$
0 \rightarrow X_{S} \rightarrow Y_{S} \rightarrow \mathbb{Z} \rightarrow 0
$$

that $\operatorname{rk}_{\mathbb{Z}}\left(Y_{S}\right)=4 r+1$. On the other hand,

$$
Y_{S}=\bigoplus_{\mathfrak{q} \in S} \mathbb{Z}[G] \otimes_{\mathbb{Z}\left[G_{\mathfrak{q}}\right]} \mathbb{Z}
$$

Hence,

$$
\begin{aligned}
4 r+1 & =\operatorname{rk}_{\mathbb{Z}}\left(Y_{S}\right) \\
& =\sum_{\mathfrak{q} \in S}\left|G: G_{\mathfrak{q}}\right| .
\end{aligned}
$$

If $G_{\mathfrak{q}}$ were strictly smaller than $G$ for all $\mathfrak{q} \in S$, then $\left|G: G_{\mathfrak{q}}\right|$ would be even for all $\mathfrak{q}$, contradicting (8.1). Therefore, there is $\mathfrak{p} \in S$ with full decomposition group. Let $\mathfrak{P}$ be the place of $L$ above $\mathfrak{p}$. Then

$$
X_{S}=\bigoplus_{\mathfrak{q} \in S \backslash\{\mathfrak{p}\}} \bigoplus_{\mathfrak{Q} \mid \mathfrak{q}} \mathbb{Z}(\mathfrak{Q}-\mathfrak{P})
$$

so since $X_{S}$ is free over $\mathbb{Z}[G], \bigoplus_{\mathfrak{Q} \mid \mathfrak{q}} \mathbb{Z}(\mathfrak{Q}-\mathfrak{P})$ is $\mathbb{Z}[G]$-projective for $\mathfrak{q} \in S \backslash\{\mathfrak{p}\}$. Since $|G|$ divides the $\mathbb{Z}$-rank of a projective module, we see that $|G|$ divides the number of places of $L$ above $\mathfrak{q}$ for each $\mathfrak{q} \in S \backslash\{\mathfrak{p}\}$. In other words, every place of $S$ other than $\mathfrak{p}$ splits completely. Counting $\mathbb{Z}$-ranks, we see that the number of such places is $r$, giving (i). We thank the referee for pointing out that (ii) implies (i).

Finally, we show that (iv) is equivalent to the other statements. That (i) implies (iv) is a consequence of [29, Chap. I, Prop. 3.4]. For the converse, we use an ad hoc argument. For $H \leq G$, let $S_{H}=\left\{v \in S \mid G_{v}=H\right\}$. Since we are assuming that $r(\chi)=r$ for all $\chi \in \widehat{G}$, [29, Chap. I, Prop. 3.4] implies that

$$
r=\# S-1
$$

(take $\chi$ to be the trivial character) and that

$$
r=\# S_{\{1\}}+\# S_{H}
$$


for each subgroup $H$ of $G$ of order 2, where $\{1\}$ is the trivial subgroup (take $\chi$ to be the unique character with kernel $H$ ). We therefore have

$$
\begin{aligned}
r & =\# S-1 \text { by }(8.2) \\
& =\# S_{G}+\# S_{\{1\}}+\sum_{H \neq\{1\}, G} \# S_{H}-1 \\
& =\# S_{G}-2 \# S_{\{1\}}+3 r-1 \text { by }(8.3),
\end{aligned}
$$

where the second equality follows because $S$ is the union of the sets $S_{H}$ as $H$ runs through the subgroups of $G$. Thus, $\# S_{\{1\}}=r+\frac{1}{2}\left(\# S_{G}-1\right)$. Therefore, $\# S_{G} \geq 1$, giving $\# S_{\{1\}} \geq r$. Since $S$ contains only $r+1$ places, we must have $\# S_{G}=1$ and $\# S_{\{1\}}=r$. In other words, $S$ contains exactly one place with full decomposition group, and the remaining $r$ places split completely. This is statement (i).

REMARK 8.2. One sees that $L / K$ admits a set $S$ satisfying the equivalent conditions of Lemma 8.1 for some $r$ if and only if the following all hold: the infinite places split completely, only one place ramifies, and the ramified place has full decomposition group.

We may now state the main theorem of the paper.

THEOREM 8.3. Let $L / K$ be a biquadratic extension in which the infinite places split completely, exactly one place ramifies, and the ramified place has full decomposition group. (See Remark 8.2.) Then there is a Rubin datum $(S, T, r)$ for $L / K$ such that $\mathrm{Z}(L / K)$ is equivalent to $\mathrm{B}(L / K, S, T, r)$.

We will see in Section 10 how to construct Rubin data $(S, T, r)$ as in the theorem.

9. Simplifying steps. Before proving the theorem, we give some preliminary results. The extension $L / K$ will be assumed to be an arbitrary abelian extension of number fields in this section, until specified further, and its Galois group will be $G$. Let $S$ be a finite set of places of $K$ containing the infinite and ramified ones, and let $T$ be a finite set of places of $K$ disjoint from $S$.

\subsection{The module $\mathbb{F}_{T}^{\times}$. Define}

$$
\mathbb{F}_{T}^{\times}=\bigoplus_{\mathfrak{p} \in T} \bigoplus_{\mathfrak{P} \mid \mathfrak{p}} \mathbb{F}_{\mathfrak{P}}^{\times},
$$

where $\mathbb{F}_{\mathfrak{P}}$ is the residue field of $\mathfrak{P}$.

LEMMA 9.1. The $\mathbb{Z}[G]$-module $\mathbb{F}_{T}^{\times}$is cohomologically trivial.

Proof. There is an exact sequence

$$
0 \rightarrow \bigoplus_{\mathfrak{p} \in T} \mathbb{Z}[G] \rightarrow \bigoplus_{\mathfrak{p} \in T} \mathbb{Z}[G] \rightarrow \mathbb{F}_{T}^{\times} \rightarrow 0 .
$$

The first map is multiplication by $1-\mathbf{N p F r}_{\mathfrak{p}}^{-1}$ in the $\mathfrak{p}$-component. (Recall that $\mathrm{Fr}_{\mathfrak{p}}$ is the arithmetic Frobenius in $G$ associated to p.) See [5, (24)] for a fuller treatment of (9.1). The lemma follows since $\mathbb{Z}[G]$ is cohomologically trivial. 
Since $\mathbb{F}_{T}^{\times}$is (finite and) cohomologically trivial, it represents a class $\left[\mathbb{F}_{T}^{\times}\right]$in $K_{0}(\mathbb{Z}[G], \mathbb{Q})$. In the description of $K_{0}(\mathbb{Z}[G], \mathbb{Q})$ as being generated by triples $(P, f, Q)$, $\left[\mathbb{F}_{T}^{\times}\right]$corresponds to the triple $(P, f, Q)$, where $P=Q=\bigoplus_{\mathfrak{p} \in T} \mathbb{Z}[G]$ and $f$ is the automorphism of $\bigoplus_{\mathfrak{p} \in T} \mathbb{Q}[G]$ described in the proof of Lemma 9.1 (extend scalars).

Since the modules in the projective resolution in (9.1) are the same, $\left[\mathbb{F}_{T}^{\times}\right]$is in the image of $K_{1}(\mathbb{Q}[G]) \rightarrow K_{0}(\mathbb{Z}[G], \mathbb{Q})$. Thus, we may view $\left[\mathbb{F}_{T}^{\times}\right]$as an element of $\mathbb{Q}[G]^{\times} / \mathbb{Z}[G]^{\times}$.

For $\chi \in \widehat{G}$, let

$$
\delta_{\chi}=L_{L / K, S, T}^{*}(0, \chi) / L_{L / K, S}^{*}(0, \chi)=\prod_{\mathfrak{p} \in T}\left(1-\chi\left(\operatorname{Fr}_{\mathfrak{p}}\right) \mathbf{N p}\right) .
$$

LEMMA 9.2. Recall that $L / K$ is an arbitrary abelian extension. The class $\left[\mathbb{F}_{T}^{\times}\right]$, viewed as an element of $\mathbb{Q}[G]^{\times} / \mathbb{Z}[G]^{\times}$, is represented by $\sum_{\chi \in \widehat{G}} \delta_{\bar{\chi}} e_{\chi}$.

Proof. From the description of the first map in (9.1), $\left[\mathbb{F}_{T}^{\times}\right]$is represented by

$$
\begin{aligned}
\prod_{\mathfrak{p} \in T}\left(1-\mathbf{N p F r}_{\mathfrak{p}}^{-1}\right) & =\sum_{\chi \in \widehat{G}} \prod_{\mathfrak{p} \in T}\left(1-\mathbf{N p F r}_{\mathfrak{p}}^{-1}\right) e_{\chi} \\
& =\sum_{\chi \in \widehat{G}} \prod_{\mathfrak{p} \in T}\left(1-\bar{\chi}\left(\mathrm{Fr}_{\mathfrak{p}}\right) \mathbf{N p}\right) e_{\chi} \\
& =\sum_{\chi \in \widehat{G}} \delta_{\bar{\chi}} e_{\chi} .
\end{aligned}
$$

Now let $\varphi: X_{S} \rightarrow U_{S}$ be an embedding of $\mathbb{Z}[G]$-modules, as in Section 5 . For a character $\chi$ of $G$, let $A_{\varphi, T}(\chi)=R_{\varphi}(\chi) / L_{L / K, S, T}^{*}(0, \chi)$. Then let $\mathcal{A}_{\varphi, T}=$ $\sum_{\chi \in \widehat{G}} A_{\varphi, T}(\bar{\chi}) e_{\chi} \in \mathbb{R}[G]^{\times}$, and let $\Upsilon_{T}(\varphi)$ be the image of $\mathcal{A}_{\varphi, T}$ in $K_{0}(\mathbb{Z}[G], \mathbb{R})$.

Lemma 9.3. Suppose $L / K$ is abelian. In $K_{0}(\mathbb{Z}[G], \mathbb{R}), \Upsilon(\varphi)-\Upsilon_{T}(\varphi)=\left[\mathbb{F}_{T}^{\times}\right]$.

Proof. By definition of the $\delta_{\chi}, \Upsilon(\varphi)-\Upsilon_{T}(\varphi)$ is the image of $\sum_{\chi \in \widehat{G}} \delta_{\bar{\chi}} e_{\chi}$ in $K_{0}(\mathbb{Z}[G], \mathbb{R})$. Now apply Lemma 9.2 .

\subsection{A simplification when $X_{S}$ is projective.}

LEMMA 9.4. Let $L / K$ be a Galois extension of number fields and $S$ a finite set of places of $K$ containing the infinite and ramified ones. Assume $\mathrm{Cl}_{S}(L)=0$. Then the following are equivalent:

(i) The Tate canonical class associated to $(L / K, S)$ is trivial.

(ii) $X_{S}$ is projective.

(iii) $U_{S}$ is cohomologically trivial.

(iv) The Tate canonical class is represented by the sequence

$$
0 \rightarrow U_{S} \stackrel{1}{\rightarrow} U_{S} \stackrel{0}{\rightarrow} X_{S} \stackrel{1}{\rightarrow} X_{S} \rightarrow 0 .
$$

Proof. Recall from (5.1) the definition of a Tate sequence. Since the modules $A$ and $B$ appearing there are cohomologically trivial, (ii) and (iii) are equivalent. (ii) implies (iv) because when $X_{S}$ is projective, $\operatorname{Ext}_{\mathbb{Z}[G]}^{2}\left(X_{S}, U_{S}\right)=0$ and so one may take 
any split sequence in which the middle modules $A$ and $B$ are cohomologically trivial and projective respectively. However, the sequence in part (iv) is such a sequence because we are assuming (ii) and (iii). Part (iv) implies (i) because the sequence in (iv) is split.

It remains to show that (i) implies (iii). Assume the Tate canonical class is trivial and choose a Tate sequence $0 \rightarrow U_{S} \rightarrow A \rightarrow B \rightarrow X_{S} \rightarrow 0$. Break it into two short exact sequences

$$
0 \rightarrow U_{S} \rightarrow A \rightarrow C \rightarrow 0
$$

and

$$
0 \rightarrow C \rightarrow B \rightarrow X_{S} \rightarrow 0
$$

Since $B$ is projective, cupping with the second of these short exact sequences induces an isomorphism $\operatorname{Ext}_{\mathbb{Z}[G]}^{1}\left(C, U_{S}\right) \rightarrow \operatorname{Ext}_{\mathbb{Z}[G]}^{2}\left(X_{S}, U_{S}\right)$. The first short exact sequence is mapped by this isomorphism to the Tate canonical class, and is therefore split because of our assumption on the Tate canonical class. Hence, $A$ is isomorphic to the direct sum of the $\mathbb{Z}[G]$-modules $U_{S}$ and $C$. Since $A$ is cohomologically trivial, so are $U_{S}$ and $C$. This establishes (iii).

In addition to the assumptions made at the beginning of Section 9, suppose that $X_{S}$ is projective and that the embedding $\varphi: X_{S} \rightarrow U_{S}$ factors through $U_{S, T}$. Consider the commutative diagram

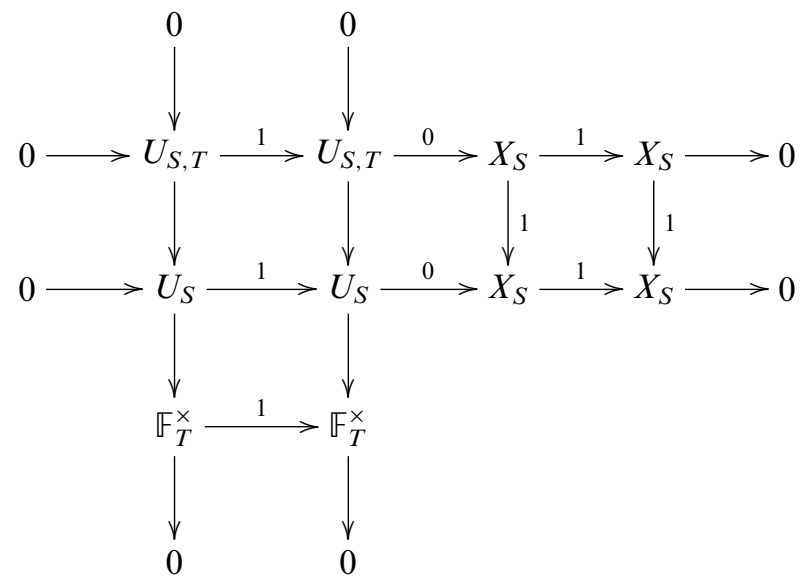

in which the rows and columns are exact. (One need not assume $X_{S}$ is projective to form a diagram of a similar kind - see $[\mathbf{5}, 7.1 .1]$. However, we make this assumption in order to deduce Lemma 9.5 below.) Since $X_{S}$ is projective, Lemma 9.4 tells us that the middle row of (9.2) is a Tate sequence for $(L / K, S)$.

Let $\Delta_{T}(\varphi, 0,0)$ be obtained by applying the construction of Section 5 to the top row of (9.2) rather than to the middle row.

LEMMA 9.5. With the foregoing assumptions (in particular, $X_{S}$ is projective), we have the following.

(i) If $\alpha, \beta$ are the maps appearing in Section 5 , then $\Xi(\alpha, \beta)=\Xi(0,0)=0$.

(ii) $\Delta(\varphi, 0,0)=\Delta_{T}(\varphi, 0,0)+\left[\mathbb{F}_{T}^{\times}\right]$.

(iii) $\omega(L / K)=\Upsilon_{T}(\varphi)-\Delta_{T}(\varphi, 0,0)$. 
Proof. (i) In Section 5, we may take $A=U_{S}, B=X_{S}$ and $C=0$. This means that the maps $\alpha$ and $\beta$ appearing there are the zero maps, and so $\Xi(\alpha, \beta)=\Xi(0,0)=0$.

(ii) This follows from the existence of (9.2) and the naturality of the construction in Section 5. See [14, p. 56 (last paragraph)].

(iii) By definition, $\omega(L / K)=\Upsilon(\varphi)-\Delta(\varphi, 0,0)+\Xi(0,0)$. However, we have just seen that $\Delta(\varphi, 0,0)=\Delta_{T}(\varphi, 0,0)+\left[\mathbb{F}_{T}^{\times}\right]$and $\Xi(0,0)=0$, and Lemma 9.3 shows that $\Upsilon(\varphi)=\Upsilon_{T}(\varphi)+\left[\mathbb{F}_{T}^{\times}\right]$.

10. Proof of Theorem 8.3. We recall the hypotheses of Theorem 8.3: $L / K$ is biquadratic, the infinite places split completely, only one place ramifies, and the ramified place has full decomposition group. Let $S_{0}$ consist of the infinite places together with the ramified place. Choose a finite set $T$ of places of $K$, disjoint from $S_{0}$, such that $U_{S_{0}, T}$ is $\mathbb{Z}$-torsion-free.

Lemma 10.1. There is a finite set $S$ of places of $K$, containing $S_{0}$, disjoint from $T$, and satisfying hypothesis $P_{r}$ for some r, such that $\mathrm{Cl}_{S}(L)$ and $\mathrm{Cl}_{S, T}(L)$ are trivial.

Proof. By [5, Lemma 5.1.1], there is a finite set $\tilde{S}$ of places of $K$, disjoint from $S_{0} \cup T$ and consisting only of places that split completely in $L / K$, such that $\mathrm{Cl}_{S_{0} \cup \tilde{S}}(L)$ and $\mathrm{Cl}_{S_{0} \cup \tilde{S}, T}(L)$ are both trivial. Since the places in $\tilde{S}$ split completely, the set $S=S_{0} \cup \tilde{S}$ satisfies hypothesis $P_{r}$ with $r=\# \tilde{S}+\# S_{0}-1$.

Let $S$ and $r$ be as in Lemma 10.1. Note that $(S, T, r)$ is a Rubin datum for $L / K$ : (St1) is satisfied trivially, (St2) and (St3) are immediate consequences of hypothesis $P_{r}$, and (St4) holds because it holds for $S_{0}$ in place of $S$ (by assumption).

10.1. Choosing the map $\varphi: X_{S} \rightarrow U_{S}$ appropriately. While the formulation of $\mathrm{Z}(L / K)$ demands no special choice of $\mathbb{Z}[G]$-embedding $X_{S} \rightarrow U_{S}$, we will choose an embedding of a particular type since it will help us in our proof of Theorem 8.3.

LEMMA 10.2. $U_{S, T} \cong X_{S}$ as $\mathbb{Z}[G]$-modules, and $U_{S}$ is cohomologically trivial. (These statements are both true in a little more generality than our current assumptions suggest; see Remark 10.3.)

Proof. We first show that $U_{S, T}$ is projective. Because of the existence of a Tate sequence for $(L / K, S)$, i.e. a sequence

$$
0 \rightarrow U_{S} \rightarrow A \rightarrow B \rightarrow X_{S} \rightarrow 0
$$

with $A$ and $B$ (finitely generated and) cohomologically trivial, the fact that $X_{S}$ is cohomologically trivial (by Lemma 8.1) implies that $U_{S}$ is also cohomologically trivial. Since $\mathrm{Cl}_{S, T}(L)$ vanishes, the five-term exact sequence in [22,(1)] yields the short exact sequence

$$
0 \rightarrow U_{S, T} \rightarrow U_{S} \rightarrow \mathbb{F}_{T}^{\times} \rightarrow 0
$$

Since $\mathbb{F}_{T}^{\times}$is cohomologically trivial by Lemma $9.1, U_{S, T}$ is cohomologically trivial as well. A $\mathbb{Z}$-torsion-free, cohomologically trivial $\mathbb{Z}[G]$-module is projective $[\mathbf{1 8}$, Prop. 1.8.4], so since $U_{S, T}$ is $\mathbb{Z}$-torsion-free by assumption, it is in fact projective. 
Now,

$$
\begin{aligned}
\mathbb{Q} \otimes_{\mathbb{Z}} U_{S, T} & \simeq \mathbb{Q} \otimes_{\mathbb{Z}} U_{S} \\
& \cong \mathbb{Q} \otimes_{\mathbb{Z}} X_{S} .
\end{aligned}
$$

Therefore, the projective modules $U_{S, T}$ and $X_{S}$ have the same $\mathbb{Z}[G]$-rank, and so their difference in $K_{0}(\mathbb{Z}[G])$ in fact lies in $\mathrm{Cl}(\mathbb{Z}[G])$. However, we saw in Lemma 6.3 that $\mathrm{Cl}(\mathbb{Z}[G])$ is trivial, so $\left[U_{S, T}\right]=\left[X_{S}\right]$ in $K_{0}(\mathbb{Z}[G])$. By Lemma 6.2, $U_{S, T} \cong X_{S}$ over $\mathbb{Z}[G]$.

REMARK 10.3. The statements in Lemma 10.2 hold for abelian extensions with the following assumptions: $X_{S}$ is projective, $\mathrm{Cl}_{S, T}(L)=0, \mathrm{Cl}(\mathbb{Z}[G])=0$ and $U_{S, T}$ is $\mathbb{Z}$-torsion-free. Indeed, these are the only facts that we have used in the proof.

Having established Lemma 10.2, we choose a $\mathbb{Z}[G]$-module isomorphism $\varphi: X_{S} \rightarrow$ $U_{S, T}$ and apply the construction of Section 5 to $\varphi$.

Proposition 10.4. With $\varphi$ chosen as above, $\omega(L / K)=\Upsilon_{T}(\varphi)$. (This holds under the assumptions discussed in Remark 10.3.)

Proof. Since $\varphi: X_{S} \rightarrow U_{S, T}$ is an isomorphism, $\Delta_{T}(\varphi, 0,0)=0$. Now use part (iii) of Lemma 9.5.

Proposition 10.5. Assume the setup from the beginning of Section 10. In particular, $L / K$ is biquadratic, and $S$ and $r$ are as in Lemma 10.1. Let $\varphi$ be as just chosen. Then $\mathrm{Z}(L / K)$ holds if and only if $A_{\varphi, T}\left(\chi_{\mathrm{reg}}\right)>0$, where $\chi_{\mathrm{reg}}$ is the character of the regular representation of $G$.

Proof. Because $G$ has only rational characters, the Strong Stark Conjecture holds for $L / K$ (recall the discussion in Section 5.1). In other words, $\omega(L / K)$ is in the kernel of $K_{0}(\mathbb{Z}[G], \mathbb{Q}) \rightarrow K_{0}(\mathcal{M}, \mathbb{Q})$, where $\mathcal{M}$ is the maximal order in $\mathbb{Q}[G]$ containing $\mathbb{Z}[G]$. Let $\omega^{\prime}(L / K)$ be the element of $\operatorname{Coker}\left(K_{1}(\mathbb{Z}[G]) \rightarrow K_{1}(\mathcal{M})\right)$ corresponding to $\omega(L / K)$, as in Lemma 6.1. By Proposition 10.4, $\omega^{\prime}(L / K)$ is represented by $\sum_{\chi \in \widehat{G}} A_{\varphi, T}(\bar{\chi}) e_{\chi}$, and so $A_{\varphi, T}(\chi) \in \mathbb{Z}^{\times}$for all $\chi \in \widehat{G}$. Now, $\omega(L / K)=0$ if and only if $\omega^{\prime}(L / K)=0$, if and only if $\sum_{\chi \in \widehat{G}} A_{\varphi, T}(\bar{\chi}) e_{\chi}$ is a unit in $\mathbb{Z}[G]$, if and only if $\left\{\chi \in \widehat{G} \mid A_{\varphi, T}(\chi)=1\right\}$ has even cardinality (by part (v) of Lemma 6.3), if and only if $\prod_{\chi \in \widehat{G}} A_{\varphi, T}(\chi)>0$, if and only if $A_{\varphi, T}\left(\chi_{\text {reg }}\right)>0$.

10.2. Connection to Rubin's Conjecture. It remains to show that $\mathrm{Z}(L / K)$ is equivalent to $\mathrm{B}(L / K, S, T, r)$. That $\mathrm{Z}(L / K)$ implies $\mathrm{B}(L / K, S, T, r)$ is given in [3, Cor. 9.2], so suppose conversely that $\mathrm{B}(L / K, S, T, r)$ holds. We claim that under our assumptions, in particular that the set $S$ satisfies hypothesis $P_{r}$, the lattice $\Omega_{S, T, r}$ is equal to $\bigwedge_{\mathbb{Z}[G]}^{r} U_{S, T}$. Indeed, $\bigwedge_{0}^{r} U_{S, T} \simeq \bigwedge_{\mathbb{Z}[G]}^{r} U_{S, T}$ by [22, Example (1), p. 38]. Further, all $\chi \in \widehat{G}$ have $r(\chi)=r$ by Lemma 8.1 , so the condition appearing in the set defining $\Omega_{S, T, r}$ is met trivially. (Recall the definition of $\Omega_{S, T, r}$ in (3.2).)

We now complete the proof of Theorem 8.3.

Proof. By Proposition 10.5, we are reduced to showing that $A_{\varphi, T}\left(\chi_{\text {reg }}\right)>0$, i.e. that the determinant of the map

$$
\mathbb{C} X_{S} \stackrel{\varphi}{\rightarrow} \mathbb{C} U_{S, T} \stackrel{\lambda}{\rightarrow} \mathbb{C} X_{S}
$$


has the same sign as $\zeta_{L, S, T}^{*}(0)$, where $\mathbb{C} M=\mathbb{C} \otimes_{\mathbb{Z}} M$ for a $\mathbb{Z}$-module $M$. This is equivalent to showing that the determinant of

$$
\bigwedge_{\mathbb{C}[G]}^{r} \mathbb{C} X_{S} \stackrel{\varphi^{(r)}}{\rightarrow} \bigwedge_{\mathbb{C}[G]}^{r} \mathbb{C} U_{S, T} \stackrel{\lambda^{(r)}}{\rightarrow} \bigwedge_{\mathbb{C}[G]}^{r} \mathbb{C} X_{S}
$$

has the same sign as $\zeta_{L, S, T}^{*}(0)$, where $\varphi^{(r)}$ is the map that $\varphi$ induces on $r$ th exterior powers. (Recall that the analogous definition was made for $\lambda^{(r)}$ in Section 3.)

Let $\left\{x_{1}, \ldots, x_{r}\right\}$ be a free $\mathbb{Z}[G]$-basis for $X_{S}$, and let $\mathbf{x}=x_{1} \wedge \cdots \wedge x_{r} \in \bigwedge_{\mathbb{Z}[G]}^{r} X_{S}$. Then by assuming $\mathrm{B}(L / K, S, T, r)$, we know that there is an element $\epsilon$ of $\wedge_{\mathbb{Z}[G]}^{r} U_{S, T}$ such that $\lambda^{(r)}(\epsilon)=\theta \mathbf{x}$, where $\theta=\theta_{L / K, S, T}^{(r)}$. Consequently, the determinant over $\mathbb{C}[G]$ of

$$
\bigwedge_{\mathbb{C}[G]} \mathbb{C} X_{S} \stackrel{\varphi_{\epsilon}}{\rightarrow} \bigwedge_{\mathbb{C}[G]}^{r} \mathbb{C} U_{S, T} \stackrel{\lambda^{(r)}}{\rightarrow} \bigwedge_{\mathbb{C}[G]}^{r} \mathbb{C} X_{S}
$$

is equal to $\theta$, where $\varphi_{\epsilon}$ sends $\mathbf{x}$ to $\epsilon$. Therefore,

$$
\begin{aligned}
\operatorname{det}_{\mathbb{C}}\left(\lambda^{(r)} \circ \varphi_{\epsilon}\right) & =\prod_{\chi \in \widehat{G}} \chi(\theta) \\
& =\prod_{\chi \in \widehat{G}} L_{L / K, S, T}^{*}(0, \chi) \\
& =\zeta_{L, S, T}^{*}(0) \\
& =(-1)^{\# T_{L}-1} R_{L, S, T} .
\end{aligned}
$$

Here $R_{L, S, T}$ is the absolute value of the determinant of the regulator map $\lambda$ with respect to $\mathbb{Z}$-bases for $U_{S, T}$ and $X_{S}$. The equality in (10.2) is the analytical class number formula.

Now, $\lambda^{(r)} \circ \varphi^{(r)}$ has determinant $\pm R_{L, S, T}$ by definition of the regulator, so the fact that $\operatorname{det}_{\mathbb{C}}\left(\lambda^{(r)} \circ \varphi_{\epsilon}\right)=(-1)^{\# T_{L}-1} R_{L, S, T}$ implies that $\epsilon$ generates $\bigwedge_{\mathbb{Z}[G]}^{r} U_{S, T}$ over $\mathbb{Z}[G]$. Letting $\mathbf{u}=\varphi^{(r)}(\mathbf{x})$, we may therefore write $\epsilon=\alpha \mathbf{u}$ with $\alpha \in \mathbb{Z}[G]^{\times}=\{ \pm \sigma \mid \sigma \in G\}$, since $\mathbf{u}$ is a free $\mathbb{Z}[G]$-generator for $\bigwedge^{r} \mathbb{Z}[G] U_{S, T}$. Then

$$
\begin{aligned}
\operatorname{det}_{\mathbb{C}}\left(\lambda^{(r)} \circ \varphi_{\epsilon}\right) / \operatorname{det}_{\mathbb{C}}\left(\lambda^{(r)} \circ \varphi^{(r)}\right) & =\operatorname{det}_{\mathbb{C}}\left(\left(\varphi^{(r)}\right)^{-1} \circ \varphi_{\epsilon}\right) \\
& =\prod_{\chi \in \widehat{G}} \chi(\alpha) \\
& =1 .
\end{aligned}
$$

The last equality follows from part (v) of Lemma 6.3. As a result, $\operatorname{det}_{\mathbb{C}}\left(\lambda^{(r)} \circ \varphi^{(r)}\right)=$ $\operatorname{det}_{\mathbb{C}}\left(\lambda^{(r)} \circ \varphi_{\epsilon}\right)$, which is equal to $\zeta_{L, S, T}^{*}(0)$ by $(10.1)$. Therefore, $\mathrm{Z}(L / K)$ holds, completing the proof of Theorem 8.3.

\section{Examples.}

11.1. Meeting the hypotheses of Theorem 8.3. We aim to find biquadratic extensions $L / K$ in which the infinite places split completely, only one place ramifies, and the ramified place has full decomposition group. Let us spend a moment justifying the existence of such extensions. There are undoubtedly many ways to find such extensions, but here is one. Let $K$ be a number field, and suppose there are a finite place $\mathfrak{p}$ and 
a positive integer $n$ such that the ray class-group of $K \bmod \mathfrak{p}^{n}$ has 2 -rank at least 2 and the $S_{0}$-class-group of $K$ has odd order, where $S_{0}$ consists of the infinite places and $\mathfrak{p}$. Such fields can be found with a computer search. (For example, take $K=\mathbb{Q}(\alpha)$, where $\alpha^{4}+6=0$ and let $\mathfrak{p}$ be the unique place above 2 ; in this case, the ray classgroup $\bmod \mathfrak{p}^{2}$ is isomorphic to $\mathbb{Z} / 2 \mathbb{Z} \times \mathbb{Z} / 2 \mathbb{Z}$ and the $S_{0}$-class-group is trivial.) With the above assumptions, there is a biquadratic extension $L / K$, unramified outside $S_{0}$, in which the infinite places split completely. It follows that $\mathfrak{p}$ is non-split (i.e. has full decomposition group) in $L / K$, for otherwise there would be an intermediate quadratic extension $F / K$ in which $\mathfrak{p}$ were split, contradicting that $\mathrm{Cl}_{S_{0}}(K)$ has odd order.

It is also worth pointing out that examples of the above type may be chosen with $L / \mathbb{Q}$ not abelian (such as the $\alpha^{4}=-6$ example). This is important, for if $L / \mathbb{Q}$ were abelian, then $\mathrm{Z}(L / K)$ would follow from the main results of [7] and [12].

11.2. Biquadratic extensions of imaginary quadratic fields. Now let $K$ be an imaginary quadratic field and $L / K$ a biquadratic extension. Assume that only one place $\mathfrak{p}$ ramifies in $L / K$ and that $\mathfrak{p}$ is non-split. (By the above argument, such extensions exist for $K=\mathbb{Q}(\sqrt{-d})$, where $d=1,2,3,5,6,10,11,13,19,22,26,29,37,38,43, \ldots$, but other values of $d$ yield examples as well. See below.) Let $S_{0}=\{\infty, \mathfrak{p}\}$, and suppose $T$ is a finite set of places of $K$, disjoint from $S_{0}$, such that $U_{S_{0}, T}$ is $\mathbb{Z}$-torsion-free. Let $\mathfrak{P}$ be the unique place of $L$ above $\mathfrak{p}$, and fix a place $w$ of $L$ above $\infty$. Finally, let $\epsilon$ be the unique $\left(S_{0}, T\right)$-unit in $L$ whose regulator is $\theta_{L / K, S_{0}, T}^{(1)}(w-\mathfrak{P})$. As discussed in Section 3.1, Stark showed in [25] that the element $\epsilon$ exists in this situation; see also Tate's treatment [29, Chap. IV, Prop. 3.9].

Proposition 11.1. If $\epsilon \in \operatorname{Fitt}_{\mathbb{Z}[G]}\left(\mathrm{Cl}_{S_{0}, T}(L)\right) U_{S_{0}, T}$, then $\mathrm{Z}(L / K)$ holds.

Proof. Choose a finite set $S$ of places of $K$, containing $S_{0}$ and disjoint from $T$, such that all places in $S \backslash S_{0}$ split completely and both groups $\mathrm{Cl}_{S}(L)$ and $\mathrm{Cl}_{S, T}(L)$ are trivial. (This is possible: see Lemma 10.1.) Let $r=\# S-1$. Recalling from Section 3.1 that $\mathrm{B}\left(L / K, S_{0}, T, 1\right)$ holds when $K$ is imaginary quadratic [25], the result [22, Theorem 5.3 (iii)] of Rubin shows that $\mathrm{B}(L / K, S, T, r)$ also holds because of our assumption on $\epsilon$. Indeed, that result of Rubin is a general assertion that, under the right conditions, knowing Rubin's Conjecture for a particular $\operatorname{Rubin}$ datum $(\mathcal{S}, \mathcal{T}, \rho)$ allows one to conclude that it still holds after totally split places are added to $\mathcal{S}$ (and $\rho$ is increased accordingly). Now apply Theorem 8.3, observing that the present Rubin datum $(S, T, r)$ serves as the Rubin datum called $(S, T, r)$ in the statement of Theorem 8.3.

We consider some specific examples. Suppose $d=17$ or $d=34$, let $K=\mathbb{Q}(\sqrt{-d})$, let $\mathfrak{p}$ be the place of $K$ above 2, and let $S_{0}=\{\infty, \mathfrak{p}\}$. The ray class field mod $\mathfrak{p}^{5}$ contains two biquadratic extensions of $K$ that are $D_{8}$-extensions of $\mathbb{Q}$. Take $L$ to be either of these extensions. The conjecture $\mathrm{Z}(L / K)$ is not known in this situation, but by Proposition 11.1, it is implied by the condition $\epsilon \in \mathrm{Fitt}_{\mathbb{Z}[G]}\left(\mathrm{Cl}_{S_{0}, T}(L)\right) U_{S_{0}, T}$. (The list of integers $d$ appearing before the statement of Proposition 11.1 is also a source of examples, but we choose $d=17,34$ to provide examples in which $L / \mathbb{Q}$ is non-abelian and $\mathrm{Cl}_{S_{0}}(L)$ is nontrivial.)

11.3. Concrete cases of $Z(L / K)$ not covered by work of Bley [1]. We finish with some concrete examples for which it is easy to deduce the truth of $\mathrm{Z}(L / K)$ from 
Theorem 8.3 with a small amount of computer calculation. It is important to note that in these examples, the field $L$ is not abelian over $\mathbb{Q}$. Further, while the base field $K$ is an imaginary quadratic field in each example, the truth of $\mathrm{Z}(L / K)$ does not follow from the work of Bley [1] mentioned in Section 3.1, since $[L: K]$ is assumed to be odd in [1].

Suppose $K=\mathbb{Q}(\alpha)$, where $\alpha$ is a root of $x^{2}+1$, let $\mathfrak{p}$ be the unique place of $K$ above 2 and let $L$ be the unique quartic abelian extension of $K$ of conductor $\mathfrak{p}^{5}$. Then $L / K$ is biquadratic, $\mathfrak{p}$ is non-split in $L / K$ and $L / K$ is unramified outside $S_{0}=\{\infty, \mathfrak{p}\}$. The class-group $\mathrm{Cl}_{S_{0}}(L)$ is trivial, and if $T=\left\{\mathfrak{q}_{1}, \mathfrak{q}_{2}\right\}$, where $\mathfrak{q}_{1}$ and $\mathfrak{q}_{2}$ are places of $K$ above 3 and 5 respectively, then $\mathrm{Cl}_{S_{0}, T}(L)$ is also trivial. The hypothesis on $\epsilon$ in Proposition 11.1 is met trivially, so $\mathrm{Z}(L / K)$ holds.

Consider instead the following example. Let $K=\mathbb{Q}(\alpha)$ and $L=K(\beta)$, where $\alpha$ is a root of $x^{2}+2$ and $\beta$ is a root of $x^{4}-8$. Then $L / K$ is biquadratic, being the compositum of the distinct quadratic extensions $K(\sqrt{\alpha}) / K$ and $K(\sqrt{-\alpha}) / K$. We again let $\mathfrak{p}$ be the place of $K$ above 2 and $S_{0}=\{\infty, \mathfrak{p}\}$. We choose the places $\mathfrak{q}_{1}$ and $\mathfrak{q}_{2}$ of $K$ (in the notation of the previous example) to lie above 3 and 17 respectively. Then both $\mathrm{Cl}_{S_{0}}(L)$ and $\mathrm{Cl}_{S_{0}, T}(L)$ are again trivial, and we deduce in the same way that $\mathrm{Z}(L / K)$ holds.

ACKNOWLEDGEMENTS. The author would like to thank Al Weiss for many helpful discussions and Daniel Macias Castillo and Henri Johnston for making useful remarks. The author is also indebted to the referee for making extensive suggestions for improvement. This research was supported in part by the Pacific Institute for the Mathematical Sciences.

\section{REFERENCES}

1. W. Bley, Equivariant Tamagawa number conjecture for abelian extensions of a quadratic imaginary field, Doc. Math. 11 (2006), 73-118 (electronic).

2. D. Burns, Equivariant Tamagawa numbers and Galois module theory. I, Compositio Math. 129 (2) (2001), 203-237.

3. D. Burns, Congruences between derivatives of abelian $L$-functions at $s=0$. Invent. Math. 169 (3) (2007), 451-499.

4. D. Burns, Congruences between derivatives of geometric $L$-functions, Invent. Math. 184 (2) (2011), 221-256 (with an appendix by Burns et al.).

5. D. Burns, On derivatives of Artin L-series, Invent. Math. 186 (2) (2011), 291-371.

6. D. Burns and M. Flach, Tamagawa numbers for motives with (non-commutative) coefficients, Doc. Math. 6 (2001), 501-570 (electronic).

7. D. Burns and C. Greither, On the equivariant Tamagawa number conjecture for Tate motives, Invent. Math. 153 (2) (2003), 303-359.

8. T. Chinburg, On the Galois structure of algebraic integers and S-units, Invent. Math. 74 (3) (1983), 321-349.

9. C. W. Curtis and I. Reiner, Methods of representation theory, vol. II. Pure and applied mathematics (with applications to finite groups and orders) (John Wiley, New York, NY, 1987). A Wiley-Interscience Publication.

10. D. S. Dummit, J. W. Sands and B. Tangedal. Stark's conjecture in multi-quadratic extensions, revisited, J. Théor. Nombres Bordeaux 15 (1) (2003), 83-97. (Les XXIIèmes Journées Arithmetiques; Lille, 2001).

11. C. J. Emmons and C. D. Popescu, Special values of abelian $L$-functions at $s=0, J$. Number Theory 129 (6) (2009), 1350-1365.

12. M. Flach, On the cyclotomic main conjecture for the prime 2, J. Reine Angew. Math. 661 (2011), 1-36. 
13. C. Greither, Arithmetic annihilators and Stark-type conjectures, in Stark's conjectures: recent work and new directions, Contemporary Mathematics, vol. 358. (American Mathematical Society, Providence, RI, 2004), 55-78.

14. K. W. Gruenberg, J. Ritter and A. Weiss, A local approach to Chinburg's root number conjecture, Proc. London Math. Soc., 79 (1) (1999), 47-80.

15. H. Johnston and A. Nickel, On the equivariant Tamagawa number conjecture for Tate motives and unconditional annihilation results. arXiv:1210.8298.

16. S. Y. Kim, On the equivariant Tamagawa number conjecture for quaternion fields, $\mathrm{PhD}$ thesis (King's College London, 2003).

17. D. Macias Castillo, On higher-order Stickelberger-type theorems for multi-quadratic extensions, Int. J. Number Theory 8 (1) (2012), 95-110.

18. J. Neukirch, A. Schmidt and K. Wingberg, Grundlehren der mathematischen wissenschaften (Fundamental principles of mathematical sciences), 2nd ed., vol. 323, Cohomology of number fields (Springer-Verlag, Berlin, Germany, 2008).

19. C. D. Popescu, On a refined Stark conjecture for function fields, Compositio Math 116 (3) (1999), 321-367.

20. C. D. Popescu, Rubin's integral refinement of the abelian Stark conjecture, in Stark's conjectures: recent work and new directions, Contemporary Mathematics, vol. 358. (American Mathematical Society, Providence, RI, 2004), 1-35.

21. C. D. Popescu, Integral and $p$-adic refinements of the abelian Stark conjecture, in Arithmetic of L-functions, IAS/Park City Mathematics Series, vol. 18 (American Mathematical Society, Providence, RI, 2011), 45-101.

22. K. Rubin, A Stark conjecture "over $\mathbf{Z}$ " for abelian $L$-functions with multiple zeros, Ann. Inst. Fourier (Grenoble) 46 (1) (1996), 33-62.

23. J. W. Sands, Popescu's conjecture in multi-quadratic extensions, in Stark's conjectures: recent work and new directions, Contemporary Mathematics, vol. 358 (American Mathematical Society, Providence, RI, 2004), 127-141.

24. J.-P. Serre, Représentations linéaires des groupes finis (Hermann, Paris, France, 1967).

25. H. M. Stark, $L$-functions at $s=1$. IV. First derivatives at $s=0$, Adv. Math. 35 (3) (1980), 197-235.

26. R. G. Swan, Algebraic K-theory, Lecture Notes in Mathematics, vol. 76 (Springer-Verlag, Berlin, Germany, 1968).

27. R. G. Swan, K-theory of finite groups and orders, Lecture Notes in Mathematics, vol. 149 (Springer-Verlag, Berlin, Germany, 1970).

28. J. Tate, The cohomology groups of tori in finite Galois extensions of number fields, Nagoya Math. J. 27 (1966), 709-719.

29. J. Tate, Les conjectures de Stark sur les fonctions $L$ d'Artin en $s=0$, Progress in Mathematics, vol. 47 (Dominique Bernardi and Norbert Schappacher, Editors) (Birkhäuser, Boston, MA, 1984).

30. D. Vallières, The equivariant Tamagawa number conjecture and the extended abelian Stark conjecture (preprint, 2012).

31. C. A. Weibel, An introduction to homological algebra, Cambridge Studies in Advanced Mathematics, vol. 38 (Cambridge University Press, Cambridge, UK, 1994).

32. A. Weiss, Multiplicative Galois module structure, Fields Institute Monographs, vol. 5 (American Mathematical Society, Providence, RI, 1996). 